\title{
ALL SOLUTIONS OF THE STOCHASTIC FIXED POINT EQUATION OF THE QUICKSORT PROCESS
}

\author{
BY S. HALLMANN, U. RÖSLER AND M. WNUK
}

\begin{abstract}
The Quicksort process $R$ (Rösler (2018)) can be characterized as the unique endogenous solution of the inhomogeneous stochastic fixed point equation $R \stackrel{\mathrm{D}}{=}\left(U R_{1}(1 \wedge t / U)+\right.$ $\left.\mathbf{1}_{\{U<t\}}(1-U) R_{2}((t-U) /(1-U))+C(U, t)\right)_{t}$ on the space $\mathscr{D}$ of càdlàg functions, such that $R(1)$ has the Quicksort distribution. In this paper we characterize all $\mathscr{D}$-valued solutions of that equation. Every solution can be represented as the convolution of a solution of the inhomogeneous equation and a general solution of the homogeneous equation (Rüschendorf (2006)). The general solutions of the homogeneous equation are the distributions of Cauchy processes $Y$ with constant drift. Any distribution of $R+Y$ for independent $R$ and $Y$ is a solution of the inhomogeneous equation. Every solution of the inhomogeneous equation is of the form $R+Y$, where $R$ and $Y$ are independent. The endogenous solutions for the inhomogeneous equation are the shifted Quicksort process distributions. In comparison, the Quicksort distribution is the endogenous solution of the Quicksort fixed point equation unique up to a constant (Rösler (1991)). The general solution can be represented as the convolution of the shifted Quicksort distribution and some symmetric Cauchy distribution (Fill and Janson (2000)), possibly degenerate.
\end{abstract}

Keywords: Stochastic process; stochastic fixed point equation; Quicksort process; Skorokhod convergence

2010 Mathematics Subject Classification: Primary 60F17

Secondary 60K99; 68P10

\section{Introduction}

One of the early results in branching processes is the convergence of the positive martingale $Z_{n} / m^{n}\left(Z_{n}\right.$ is a Bienaymé-Galton-Watson process and $\left.m=\mathbb{E}\left[Z_{1}\right]\right)$ to a limit $W$ that satisfies the equation

$$
W=\sum_{i \in \mathbb{N}} \frac{\mathbf{1}_{\{X \geq i\}}}{m} W_{i}
$$

with appropriate notation [4]. The limit $W$ is endogenous, i.e. measurable with respect to the natural filtration generated by the offspring. In this setting we consider the branching process starting with one particle at time 0 and proceed forwards in time. Running the time forwards seems very natural in view of biological examples, evolution of individuals, and daily life experience.

For the construction of phylogenetic trees, we know the present and try to run the branching process backwards in time in order to recover the whole genetic tree. In the limit we have an entrance boundary at infinity and run the branching process backwards in time. By this intuition, we obtain more solutions of the above equation, a whole bunch of nonendogenous solutions [2]. 
They are nonendogenous, since at infinity some random variables (i.e. information) are added, and they cannot be recovered from the observed process.

Consider now a weighted branching process (WBP) [22]. Name the vertices (i.e. individuals) $v$ in Ulam-Harris notation, while the edges $(v, v i)$ are equipped with some transformations $T_{v, v i}$. These transformations transport a weight either forwards from the individual $v$ to $v i$ or backwards from the individual $v i$ to $v$. We call this object a WBP if $\left(T_{v, v i}\right)_{i \in \mathbb{N}}, v \in V$, are all independent and identically distributed random variables. Note that we require independence of the families, but allow arbitrary dependence within a family. To every path $(v, v w)$ we assign a transformation $L_{v, v w}$ by following the path from $v$ to $v w$ and composing the $T$-transformations we pass. If we add some cost (toll) function $C_{v}$ for passing the vertex $v$ then we require $\left(C_{v},\left(T_{v, v i}\right)_{i \in \mathbb{N}}\right), v \in V$, to be independent, identically distributed random variables.

Again we seek solutions to either the homogeneous equation

$$
W=\sum_{i \in \mathbb{N}} T_{i}\left(W_{i}\right)
$$

or the inhomogeneous equation

$$
W=\sum_{i \in \mathbb{N}} T_{i}\left(W_{i}\right)+C .
$$

Here $\left(C,\left(T_{i}\right)_{i}\right), W_{j}, j \in \mathbb{N}$, are mutually independent and the $W_{j}$ have the same distribution as $W$. Equivalently, we can seek solutions to the stochastic fixed point equations (SFPEs)

$$
W \stackrel{\mathrm{D}}{=} \sum_{i \in \mathbb{N}} T_{i}\left(W_{i}\right) \quad \text { and } \quad W \stackrel{\mathrm{D}}{=} \sum_{i \in \mathbb{N}} T_{i}\left(W_{i}\right)+C .
$$

There exist many examples of such recursive structures [1], [24], [25], especially in the (running time) analysis of algorithms, using the contraction method [16], [17], [24], which provides endogenous solutions.

It is a more demanding mathematical question to find all solutions of SFPEs. We know the set of all real-valued solutions of an SFPE if the dynamic is multiplication and the $T$-random variables are either degenerate and real [3], positive real [11], [10], or real [2]. Furthermore, there are some results for solutions in $R^{d}$ [15] and for complex-valued solutions [14], but only few for $\mathcal{D}$-valued processes [17].

We are interested in $\mathscr{D}$-valued processes [5]. In fact, we consider processes $W=\left(W_{t}\right)_{t \in[0,1]}$ with paths in the space $\mathscr{D}_{-}$of càglàd (continue à gauche, limite à droite, i.e. left continuous with existing right limits) functions $f:[0,1] \rightarrow \mathbb{R}$ with the convention that $f(0)=0$. The interpretation and notation of the processes is more natural in $\mathscr{D}_{-}$than in $\mathscr{D}$. Mathematically, it makes no difference to work here with càglàd or càdlàg functions.

The standard examples are Brownian motion and some Lévy processes, where $T$. are spacetime transformations. To give just one example, standard Brownian motion satisfies

$$
W \stackrel{\mathrm{D}}{=}\left(\sqrt{U} W_{1}\left(1 \wedge \frac{t}{U}\right)+\sqrt{1-U} W_{2}\left(0 \vee \frac{t-U}{1-U}\right)\right)_{t}
$$

for $U$ a $(0,1)$-valued random variable that is independent of $W_{1}$ and $W_{2}$.

Two more examples occur in the running time analysis of random algorithms. The Quickselect (or Find) process [7], [8], [9] is characterized by the SFPE

$$
W \stackrel{\mathrm{D}}{=}\left(\mathbf{1}_{\{t \leq U\}} U W_{1}\left(\frac{t}{U}\right)+\mathbf{1}_{\{t>U\}}(1-U) W_{2}\left(\frac{t-U}{1-U}\right)+1\right)_{t},
$$


where $U$ is uniformly distributed on $(0,1)$. (The Find-algorithm searches for the $\ell$ th smallest element out of $n$. Let $X(n, \ell)$ be the number of comparisons. Then $Y_{n}$ defined by $Y_{n}(\ell / n)=$ $X(n, \ell) / n$ as a process on $[0,1]$ converges in distribution (or in Skorokhod metric for nice versions) to a solution of the above SFPE.) In this case we have more a perpetuity than real branching.

The other example is recent; the Quicksort process [23], satisfying the SFPE

$$
W \stackrel{\mathrm{D}}{=}\left(U W_{1}\left(1 \wedge \frac{t}{U}\right)+(1-U) W_{2}\left(0 \vee \frac{t-U}{1-U}\right)+C(U, t)\right)_{t} .
$$

The random variables $W_{1}, W_{2}$, and $U$ are independent, $W_{1}$ and $W_{2}$ have the same distribution as $W$, and $U$ is uniformly distributed on the unit interval. The function $C:[0,1] \times(0,1] \rightarrow \mathbb{R}$ is given by

$$
C(s, t):=\mathbf{1}_{\{t>s\}} C(s)+\mathbf{1}_{\{t \leq s\}}[-1+2 s+2(1-t) \ln (1-t)-2(s-t) \ln (s-t)+2 s \ln s]
$$

and

$$
C(s):=1+2 s \ln s+2(1-s) \ln (1-s),
$$

using continuous extension. We extend $C$ by $C(\cdot, 0)=0$.

The Quicksort process $R$ appears as the limit [18], [23] of the normalized running time of some version of the algorithm Partial Quicksort, introduced by Martínez [12], [13]. The proof makes considerable use of embedding the algorithm into a WBP and simultaneously using the forwards and backwards views of the WBP.

In this paper we are interested in all solutions of SFPE (1.1) in $\mathscr{D}_{-}$(respectively $\left.\mathscr{D}\right)$. The Quicksort process $R$ is the only endogenous [1] (measurable with respect to the random variables $T$ and $C$ of the corresponding WBP) solution of SFPE (1.1) such that $R(1)$ has the Quicksort distribution. Other endogenous solutions are $R+b I$, where $I$ is the identity, and these are all. Any solution with finite first moment of the process at some time $t \in(0,1]$ (and then for any $t \in(0,1])$ is endogenous and vice versa.

Theorem 1.1. Any solution of SFPE(1.1) in $D_{-}$has a unique representation as the distribution on $D_{-}$of $R+a Y+b I$, where $a, b \in \mathbb{R}, a \geq 0, R$ is the Quicksort process, $Y$ is an independent standard Cauchy process, and I denotes the identity.

The following outline of the proof precedes a formal proof given in Section 3. The easy part is to show that the above processes are solutions; the difficult part is to show that there are no other solutions. Evaluating (1.1) at the time point $t=1$ yields the real-valued Quicksort SFPE [20], [21], $Q \stackrel{\mathrm{D}}{=} W(1)$,

$$
Q \stackrel{\mathrm{D}}{=} U Q_{1}+(1-U) Q_{2}+C(U)
$$

where $C$ is given in (1.2). Fortunately, by [2] and [6], we know all solutions of SFPEs

$$
W \stackrel{\mathrm{D}}{=} \sum_{i \in \mathbb{N}} T_{i} W_{i}+C
$$

where $\left(C,\left(T_{i}\right)_{i \in \mathbb{N}}\right), W_{j}, j \in \mathbb{N}$, are independent random variables, the $W_{j}$ are distributed as $W$, and all random variables are real valued. Under some very general conditions, the solutions can be represented as the distribution of a solution $R$ of the inhomogeneous SFPE (1.3) and some general solution for the homogeneous SFPE ((1.3) with $C \equiv 0)$

$$
R+Z^{1 / \alpha} Y
$$


Here $Y$ is an $\alpha$-stable random variable, $Z$ is an endogenous positive solution of the SFPE $X \stackrel{\mathrm{D}}{=} \sum_{i}\left|T_{i}\right|^{\alpha} X_{i}$, and $\alpha$ is such that $\mathbb{E}\left[\sum_{i}\left|T_{i}\right|^{\alpha}\right]=1$ and there exists $\beta>\alpha$ with $\mathbb{E}\left[\sum_{i}\left|T_{i}\right|^{\beta}\right]<1$. The random variables $(R, Z)$ and $Y$ are independent. In our case $\alpha=1$ and $Z$ is degenerate at a constant.

As noted, we have control over the distribution of $W(1)$ of the general solution of SFPE (1.1) in $\mathscr{D}_{-}$. If we replace the distributional equality in (1.1) by equality of random variables then we also control $W(t)=U W_{1}(1)$ at the random time point $t=U$. Replacing the recursive distributional equalities for $W_{1}$ and $W_{2}$ by equality of random variables, we also control $W(t)$ at some random time points in $(0, U]$ and $(U, 1]$, respectively. Iterating $(1.1)$ several times with equality of random variables we control $W$ at more and more time points. In the limit we know $W(t)$ at countably many time points $t$; these time points are dense in $(0,1]$ and determine the path of $W$ completely by continuity arguments. It remains to show that there is a version of $W_{v}, U_{v}, v \in V$, such that

$$
W_{v}=\left(U_{v} W_{v 1}\left(1 \wedge \frac{t}{U_{v}}\right)+\left(1-U_{v}\right) W_{v 2}\left(0 \vee \frac{t-U_{v}}{1-U_{v}}\right)+C\left(U_{v}, t\right)\right)_{t}
$$

holds simultaneously for all $v \in V$. This follows from Kolmogorov's result on projective limits for consistent probability spaces (see [19]).

Our motivation for this study came from the algorithm Partial Quicksort introduced by Martínez [12]. The input is a list of $n$ different real numbers for $n \geq 2$. The output of Partial Quicksort for given $0 \leq k<\ell \leq n$ is the sorted list of all numbers between the $k$ th and $\ell$ th smallest numbers of the input. We present here the deterministic algorithm Quicksort on the fly given any finite input list. It works as follows:

- always choose the first element in the list as the pivot;

- sequentially compare every other element in the list with the pivot and thereby form three smaller lists: the first list consists of all elements smaller than the pivot, the second consists of the pivot, and the third consists of all elements larger than the pivot, though it is possible for the first or last list to be null; and

- repeat from the first step above using the leftmost list with at least two elements.

Given $n$ different real numbers, after applying these three steps $k$ times there are between $2 k$ and $\min \{n, 3 k\}$ nonempty 'lists' of which at least $k$ contain one element and the largest has at most $n-k$ elements. The algorithm terminates when the collection consists of $n$ 'lists', each consisting of just one real number.

If we stop the algorithm Quicksort on the fly when the $\ell$ th smallest element is identified then we will have performed a version of the algorithm Partial Quicksort, namely, find the $\ell$ th smallest numbers ordered out of $n$, and we will have performed only the necessary comparisons and not more.

We now consider $\ell / n$ as some time index and take the limit $n \rightarrow \infty$. Let $X(u, \ell)$ denote the number of comparisons necessary to find and sort the $0 \leq \ell \leq|u| \in \mathbb{N}_{0}$ smallest numbers given a finite input $u$. The Quicksort on the fly recursion satisfies

$$
X(u, \ell)=X\left(u^{1}, l \wedge\left|u^{1}\right|\right)+X\left(u^{2}, 0 \vee\left(\ell-\left|u^{1}\right|-1\right)\right)+|u|-1
$$

for $1 \leq \ell \leq|u|,|u| \geq 2$, according to the above splitting procedure. In (1.5), $u^{1}$ and $u^{2}$ denote the lists for $2 \leq i \leq|u|$ of all $u_{i}<u_{1}$ and $u_{i}>u_{1}$, respectively. 
Let $U=\left(U_{n}\right)_{n \in \mathbb{N}}$ be a vector of independent random variables with a uniform distribution on the open unit interval, and let $U_{\mid n}:=\left(U_{1}, U_{2}, \ldots, U_{n}\right)$. Then the distribution of the process $\left(X\left(U_{\mid n}, \ell\right)\right)_{\ell}$ as in (1.5) depends only on the size $n$ of the input. We obtain the following recursion for the distribution of $X(n, \cdot)$ for $n \geq 2$ :

$$
(X(n, \ell))_{\ell} \stackrel{\mathrm{D}}{=}\left(n-1+X_{1}(I-1, \ell \wedge(I-1))+X_{2}(n-I, 0 \vee(\ell-I))\right)_{\ell} .
$$

Here, for given $n$, the random variables $I$ and $X_{i}(j, \cdot), i=1,2, j<n$, are independent. The random variable $I=I_{n}$ is uniformly distributed on $\{1,2, \ldots, n\}$ and $X_{i}(j, \cdot)$ has the same distribution as $X(j, \cdot)$. We use the same starting values $X(n, 0)=0=X(1,1)$ as above. The random variable $I$ has the interpretation of the rank of the pivot $U_{1}$ within $U_{\mid n}$.

The expectation $\mathbb{E}[X(n, \ell)]=: a(n, \ell)$ was given explicitly by Martínez [12] as

$$
a(n, \ell)=2 n+2(n+1) H_{n}+2(n+3+\ell) H_{n+1+\ell}-6 \ell+6, \quad 1 \leq \ell \leq n,
$$

where $H_{k}=\sum_{i=1}^{k} 1 / i$ denotes the harmonic number.

The distributions of

$$
\left(Y\left(n, \frac{\ell}{n}\right)\right)_{\ell}=\left(\frac{X(n, \ell)-a(n, \ell)}{n+1}\right)_{\ell}
$$

satisfy the recursions for process distributions for $n \geq 2$, i.e.

$$
\begin{aligned}
\left(Y\left(n, \frac{\ell}{n}\right)\right)_{\ell} \stackrel{\mathrm{D}}{=} & \left(\frac{I}{n+1} Y_{1}\left(I-1,1 \wedge \frac{\ell}{I-1}\right)+\frac{n+1-I}{n+1} Y_{2}\left(n-I, 0 \vee \frac{\ell-I}{n-I}\right)\right. \\
& \left.+\frac{C(n, I, \cdot)}{n+1}\right)_{\ell},
\end{aligned}
$$

where $I=I_{n}$ is as above and

$$
C(n, i, l)=n-1-a(n, l)+a(i-1, l \wedge(i-1))+a(n-i, 0 \vee(l-i)) .
$$

The distribution of $Y(n, 1)$ is called the discrete Quicksort distribution with parameter $n$ (to sort $n$ elements).

Martínez and Rösler [13] proved the weak convergence of $Y\left(n, t_{n}\right)$ as $n \rightarrow \infty$ when also $t_{n} \rightarrow t$. The convergence of finite-dimensional distributions was shown by Ragab and Roesler [18]. The limit is the Quicksort process in $\mathscr{D}_{-}$(or $\left.\mathscr{D}\right)$ satisfying (1.1). The given specific processes $Y\left(U_{\mid n}, \cdot\right)$ even converge in the Skorokhod metric to the (right version of the) Quicksort process pointwise and uniformly almost everywhere (a.e.) as $n \rightarrow \infty$ [23].

\section{Recursive equations}

In this section we show the existence of the required version satisfying (1.4).

We use the Ulam-Harris notation for a binary tree $V:=\{1,2\}^{*}:=\bigcup_{n \in \mathbb{N}_{0}}\{1,2\}^{n}$, where $\mathbb{N}_{0}=\mathbb{N} \cup\{0\}$ and $\{1,2\}^{0}=\{\varnothing\}$. Let $V_{m}=\{1,2\}^{m},\left(V_{<m}\right)$, denote the set of $v \in V$ with length $m$ (or strictly smaller than $m$ ). We consider $V$ as a directed graph with edges $(v, v i), v \in V, i \in\{1,2\}$. We take $e_{v}=\{(v, v 1),(v, v 2)\}$ as the set of all edges starting in $v \in V$, and let $E$ denote all the sets $e_{v}, v \in V$. We use $E_{<m}$ for the subset of all $e_{v}, v \in V_{<m}$.

Let $\left(\mathscr{D}_{-}, \mathscr{B}_{\mathscr{D}}, \rho\right)$ be the space of càglàd functions with value 0 at 0 , endowed with the Borel $\sigma$-field $\mathscr{B}_{\mathscr{D}}$, and let $\rho$ be the probability measure of a solution to SFPE (1.1). Take the Borel 
$\sigma$-field $\mathscr{B}$ on the squared unit interval $(0,1)^{2}$ and the probability measure $v$ on $\left((0,1)^{2}, \mathscr{B}\right)$ defined by $v(A \times B)=\lambda(A \cap(1-B))$ for the one-dimensional Lebesgue measure $\lambda$ on $(0,1)$.

Let

$$
\left(\Omega_{i}, \mathcal{A}_{i}, \mu_{i}\right):= \begin{cases}\left(\mathscr{D}_{-}, \mathcal{B}_{\mathscr{D}}, \rho\right) & \text { if } i \in V, \\ \left((0,1)^{2}, \mathscr{B}, \nu\right) & \text { if } i \in E .\end{cases}
$$

For a set $J \subset V \cup E$, define the product probability space

$$
\left(\Omega_{J}, \mathcal{A}_{J}, \mu_{J}\right):=\left(\prod_{j \in J} \Omega_{j}, \bigotimes_{j \in J} \mathcal{A}_{j}, \bigotimes_{j \in J} \mu_{j}\right)
$$

with the product $\sigma$-field and product measure, and for $K \subset J \subset V \cup E$, denote projections by $\Phi_{J, K}: \Omega_{J} \rightarrow \Omega_{K}$.

For $I_{m}:=V_{m} \cup E_{<m}, m \in \mathbb{N}$, we use the short form $\left(\Omega_{m}, \mathcal{A}_{m}, \mu_{m}\right)$ for the product measure space. Let $\bar{I}_{m}:=V_{\leq m} \cup E_{<m}$ for $m \in \mathbb{N}$. On the product measurable space $\left(\bar{\Omega}_{m}, \overline{\mathcal{A}}_{m}\right)=$ $\left(\Omega_{\bar{I}_{m}}, \mathcal{A}_{I_{m}}\right)$ introduce the probability measure $\bar{\mu}_{m}$. We specify the measure on $\bar{\Omega}_{m}$ by $\bar{\mu}_{m}\left(\Phi_{\bar{I}_{m}, I_{m}}^{-1}(\cdot)\right)=\mu_{m}(\cdot)$, and let the other values of the coordinates be determined by the given values for $\Omega_{m}$ and the recursions of (1.3) and (1.4), replacing equality in distribution by equality for random variables, respectively. Formally, let $h: D_{-} \times D_{-} \times(0,1)^{2} \rightarrow D_{-}$be defined by

$$
h(f, g, x, y)(t)=x f\left(1 \wedge \frac{t}{x}\right)+y g\left(0 \vee \frac{t-y}{1-y}\right)+C(x, t) .
$$

For given values $V_{m} \ni w \mapsto X_{w}:=\Phi_{\bar{I}_{m},\{w\}} \in \mathscr{D}_{-}$and

$$
E_{<m} \ni e_{w} \mapsto\left(U_{w}, 1-U_{w}\right):=\left(\Phi_{\bar{I}_{m},\{(w, w 1)\}}, \Phi_{\bar{I}_{m},\{(w, w 2)\}}\right) \in(0,1)^{2},
$$

recursively define

$$
X_{v}=h\left(X_{v 1}, X_{v 2}, U_{v}, 1-U_{v}\right), \quad v \in V_{<m} .
$$

Lemma 2.1. The probability spaces $\left(\bar{\Omega}_{m}, \overline{\mathcal{A}}_{m}, \bar{\mu}_{m}\right), m \in \mathbb{N}$, form a projective family with respect to the projections as morphisms. A projective limit exists and can be chosen as $\left(\Omega_{V \times E}, \mathcal{A}_{V \times E}, \mu_{V \times E}\right)$ with projections as morphisms.

Proof. For consistency, it suffices to show that, for all $m \in \mathbb{N}$ and $A \in \overline{\mathcal{A}}_{m}$,

$$
\bar{\mu}_{m+1}\left(\Phi_{\bar{I}_{m+1}, \bar{I}_{m}}^{-1}(A)\right)=\bar{\mu}_{m}(A) \text {. }
$$

Fix $m$ and, for brevity, write $X_{v}=\Phi_{\bar{I}_{m},\{v\}}$ for $v \in V_{\leq m}$ and $U_{w}=\Phi_{\bar{I}_{m+1},\{(w, w 1)\}}$ for $w \in V_{\leq m}$. Then

$$
X_{v}=h\left(X_{v 1}, X_{v 2}, U_{v}, 1-U_{v}\right) \text { for all } v \in V_{m} .
$$

For consistency, it suffices to prove the independence of $X_{v}, v \in V_{m}$, and that the distribution of every $X_{v}, v \in V_{m}$, under $\bar{\mu}_{m+1}$ is $\rho$. Independence is obvious from the construction via a product measure $\mu_{m+1}$, and the distribution is the correct distribution because $\rho$ satisfies the SFPE.

All $\Omega$. are Polish spaces endowed with the Borel $\sigma$-algebra. From Kolmogorov's result on projective families we obtain a projective limit, and this limit can be represented on the product measurable space with a suitable probability measure. 


\section{Solutions}

A Cauchy distribution $\operatorname{Cau}(b, c)$ on the reals for $c, b \in \mathbb{R}, c>0$, is determined by the characteristic function $\mathbb{R} \ni \lambda \rightarrow \mathrm{e}^{b \lambda-c|\lambda|}$. A symmetric Cauchy distribution has the parameters $b=0$ and $c>0$. Every Cauchy random variable with distribution $\mathrm{Cau}(b, c)$ has a representation $X+b$, where $X$ has a $\mathrm{Cau}(0, c)$ distribution. The standard Cauchy distribution has the pair of parameters $(0,1)$ and is symmetric.

Lemma 3.1. If the homogeneous SFPE

$$
W \stackrel{\mathrm{D}}{=}\left(U W_{1}\left(1 \wedge \frac{t}{U}\right)+(1-U) W_{2}\left(0 \vee \frac{t-U}{1-U}\right)\right)_{t}
$$

in D_ has a solution for which W(1) is standard Cauchy distributed, then the solution is unique.

Proof. We use the same construction as in Lemma 2.1. Let $W_{v}=\Phi_{V \times E,\{v\}}$ for $v \in V$ and $\left(U_{v}, 1-U_{v}\right)=\Phi_{V \times E,\left\{e_{v}\right\}}$ for $e_{v} \in E$.

Let $H$ be the set of half-open intervals $(c, d]$ in the unit interval. Let $G$ be the subspace of $H^{H}$ generated by the maps $g_{v, v i}: H \rightarrow H$ for which

$$
g_{v, v 1}((c, d])=\left(c,(d-c) U_{v}\right], \quad g_{v, v 2}=\left((d-c) U_{v}, d\right],
$$

to which we add the identity and a cemetery state. ( $\varnothing$ plays the role of a cemetery state in $H$ and the function identically $\varnothing$ in $G$ is the identity.) $G$ is a semigroup $(G, *)$ with composition $g * \bar{g}=\bar{g} \circ g$.

Define the path weights $L$ by $L_{v, v i}=g_{v, v i}$ and recursively by $L_{v, v w} * L_{v w, v w x}=L_{v, v w x}$. Denote the interval $L_{\varnothing, v}((0,1])$ by $J_{v}=\left(c_{v}, d_{v}\right], v \in V$. The $J_{v}, v \in V_{m}$, form a partition of $(0,1]$ for every $m \in \mathbb{N}$. Note that the random sets $\left\{c_{v} \mid v \in V_{m}\right\}$ and $\left\{d_{v} \mid v \in V_{m}\right\}$ are nested in $m$, and converge to a countable dense random set.

Iterating (3.1) with equality of the random variables $m$-times we obtain

$$
W=\left(\sum_{v \in V_{m}}\left(d_{v}-c_{v}\right) W_{v}\left(0 \vee \frac{t-c_{v}}{d_{v}-c_{v}} \wedge 1\right)\right)_{t} .
$$

Note that we assumed $W$.(1) to be standard Cauchy. For fixed $m$ and $t \in(0,1]$, we observe that

$$
\begin{aligned}
W(t) & =\left(\sum_{V_{m} \ni v \prec_{l} w_{m, t}}\left(d_{v}-c_{v}\right) W_{v}(1)\right)+\left(d_{w_{m, t}}-c_{w_{m, t}}\right) W_{w_{m, t}}\left(\frac{t-c_{w_{m, t}}}{d_{w_{m, t}}-c_{w_{m, t}}}\right) \\
& =: I+I I .
\end{aligned}
$$

Here $w_{m, t}$ is the element $w \in V_{m}$ such that $t \in J_{w}$, and the sum is over all $v \in V_{m}$ that are strictly smaller than $w_{m, t}$ in lexicographic order $\left(\preceq_{1}\right)$.

For $t \in(0,1]$, the distribution of $W(t)$ is $\operatorname{Cau}(0, t)$. The first term of $I$ is $W\left(c_{w_{m, t}}\right)$ by construction. From the independence of the $W(v), v \in V_{m}$, for given $c$. and $d$. which are $\left(U_{w}\right)_{w \in V}$ measurable, we conclude that $W\left(c_{w_{m, t}}\right)$ has a symmetric Cauchy distribution with the (random) parameter $\left(0, c_{w_{m, t}}\right)$. For fixed $t$ and $m \rightarrow \infty$, this parameter converges to $(0, t]$ almost surely (a.s.). From the left continuity of $W$ and $0 \leq t-c_{w_{m, t}} \searrow m 0$ a.s., we deduce that, as $m \rightarrow \infty, W(t)-W\left(c_{w_{m, t}}\right)$ converges to 0 a.e.

The finite marginal distributions of $W$ are those of a standard Cauchy process. Let $0=$ $t_{0}<t_{1}<\cdots<t_{n}<t_{n+1}=1$ be in $[0,1]$. We are interested in the distribution of $\left(W\left(t_{i}\right)-W\left(t_{i-1}\right)\right)_{i=1}^{n+1}$. For large $m$, we may assume that every $t_{i}, 1 \leq i \leq n$, is in some interval $J_{v^{m, i}}=\left(c_{v^{m, i}}, d_{v^{m, i}}\right]$ for different $v^{m, i} \in V_{m}$. Note that $c_{v^{m, i}}$ is increasing in $m, d_{v^{m, i}}$ is 
decreasing in $m$, and both converge as $m \rightarrow \infty$ to $t_{i}$ a.e. Consider the vector of differences

$$
\left(W\left(c_{v^{m, 1}}\right)-0, W\left(c_{v^{m, 2}}\right)-W\left(d_{v^{m, 1}}+\right), \ldots, W\left(c_{v^{m, n}}\right)-W\left(d_{v^{m, n-1}}+\right), 1-W\left(d_{v^{m, n}}+\right)\right) .
$$

Every coordinate is the sum over some terms of the form $\left(d_{v}-c_{v}\right) W_{v}(1), v \in V_{m}$. By independence of the $W_{v}, v \in V_{m}$, and conditioned on $\left(U_{w}\right)_{w \in V}$ (note that the $c$. and $d$. values are $\left(U_{w}\right)_{w}$ measurable), the difference vector above is

$$
\left(\mathrm{Cau}\left(0, c_{v^{m, 1}}\right), \mathrm{Cau}\left(0, c_{v^{m, 2}}-d_{v^{m, 1}}\right), \ldots, \mathrm{Cau}\left(0, c_{v^{m, n}}-d_{v^{m, n-1}}\right), \mathrm{Cau}\left(0,1-d_{v^{m, n}}\right)\right)
$$

distributed. If we let $m$ converge to $\infty$ then the distribution of the difference vector converges to

$$
\left(\mathrm{Cau}\left(0, t_{1}\right), \operatorname{Cau}\left(0, t_{2}-t_{1}\right), \ldots, \mathrm{Cau}\left(0, t_{n}-t_{n-1}\right), \operatorname{Cau}\left(0,1-t_{n}\right)\right) .
$$

From the pointwise almost-sure convergence of the $c$. and $d$., we conclude that the $W$-difference vector converges to

$$
\left(W\left(t_{1}\right)-0, W\left(t_{2}\right)-W\left(t_{1}+\right), \ldots, W\left(t_{n}\right)-W\left(t_{n-1}+\right), W(1)-W\left(t_{n}+\right)\right)
$$

pointwise a.s., and, therefore, also in distribution. Finally, use $\mathbb{P}(W(t) \neq W(t-))=0$ for every $t \in[0,1]$ to delete the right limits in this last vector.

The standard Cauchy distribution is a solution in the sense of Lemma 3.1. For every solution, any multiple is a solution. Also, the deterministic function $W(t)=b t$ for all $t$ is a solution.

Proof of Theorem 1.1. We first show that $R+a Y+b I$ is a solution of SFPE (1.1). Take independent copies $W_{1}=R_{1}+a Y_{1}+b I$ and $W_{2}=R_{2}+a Y_{2}+b I$ of the solution. Let $U$ be uniformly distributed, independent of the copies, and substitute the values into the right-hand side of the Quicksort process SFPE (1.1). We obtain

$$
\begin{aligned}
& \left(\left[U\left(R_{1}+b\right)\left(1 \wedge \frac{t}{U}\right)+(1-U)\left(R_{2}+b\right)\left(0 \vee \frac{t-U}{1-U}\right)+C(U, t)\right]\right. \\
& \left.+\left[a U Y_{1}\left(1 \wedge \frac{t}{U}\right)+a(1-U) Y_{2}\left(0 \vee \frac{t-U}{1-U}\right)\right]\right)_{t} \\
& \quad=: I+I I .
\end{aligned}
$$

The distribution of $I$ is the same as that of $R+b I$. The distribution of $I I$ is the same as that of $a Y$. Argue that the Cauchy process is a strong Markov process and we can stop a Cauchy process with $U$ as the stopping time. Up to time $U$, it is a Cauchy process, and after time $U$, it is, conditioned on the past of the stopping time $U$, again an independent Cauchy process with the same parameter. Now a suitable random time change as given for the process up to the stopping time $U$ and another for the process after the stopping time makes II a Cauchy process with the right parameter.

To show the independence of $I$ and $I I$, simply observe that, for given $U$, the conditional distribution of $I I$ under $U$ is a Cauchy process with parameter that does not depend on $U$.

Finally, we show that there are no more solutions other than those we have proposed. To every solution $\rho$ construct the projective limit as given in Lemma 2.1. Take $W_{w}=\Phi_{V \times E,\{w\}}$ and $\left(U_{w}, 1-U_{w}\right)=\Phi_{V \times E,\left\{e_{w}\right\}}$ for $w \in V$. Then we obtain the equality

$$
W_{v}=\left(U_{v} W_{v 1}\left(1 \wedge \frac{t}{U_{v}}\right)+\left(1-U_{v}\right) W_{v 2}\left(0 \vee \frac{t-U_{v}}{1-U_{v}}\right)+C\left(U_{v}, t\right)\right)_{t}
$$


The endogenous solutions $R_{v}, v \in V$, for the Quicksort processes starting in $v$ satisfy [23]

$$
R_{v}=\left(U_{v} R_{v 1}\left(1 \wedge \frac{t}{U_{v}}\right)+\left(1-U_{v}\right) R_{v 2}\left(0 \vee \frac{t-U_{v}}{1-U_{v}}\right)+C\left(U_{v}, t\right)\right)_{t} .
$$

Therefore, $W_{v}-R_{v}=: Y_{v}$ satisfies the homogeneous equation

$$
Y_{v}=\left(U_{v} Y_{v 1}\left(1 \wedge \frac{t}{U_{v}}\right)+\left(1-U_{v}\right) Y_{v 2}\left(0 \vee \frac{t-U_{v}}{1-U_{v}}\right)\right)_{t} ;
$$

$Y_{v}(1)$ satisfies the equation $Y_{v}(1)=U_{v} Y_{v 1}(1)+\left(1-U_{v}\right) Y_{v 2}(1)$ in $\mathbb{R}$. We know all solutions of the corresponding fixed point equation [2]: every solution is a Cauchy distribution, possibly degenerate, with parameters $b \in \mathbb{R}$ and $c \geq 0$. The degenerate case, $c=0$, is the point measure in 0 corresponding to $a=0$ in the representation. For $c>0$, the process $(Y-b) / c$ has a standard Cauchy distribution at $t=1$. Lemma 3.1 states that there is at most one solution $Y$ of the homogeneous SFPE in $D_{-}$with $Y(1)$ standard Cauchy distributed with parameter $(0,1)$. It must be the standard Cauchy process.

\section{References}

[1] Aldous, D. J. and Bandyopadhyay, A. (2005). A survey of max-type recursive distributional equations. Ann. Appl. Prob. 15, 1047-1110.

[2] Alsmeyer, G. and Meiners, M. (2013). Fixed points of the smoothing transform: two-sided solutions. Prob. Theory Relat. Fields 155, 165-199.

[3] Alsmeyer, G. ANd Rösler, U. (2006). A stochastic fixed point equation related to weighted branching with deterministic weights. Electron. J. Prob. 11, 27-56.

[4] Athreya, K. B. And Ney, P. E. (1972). Branching Processes (Die Grundlehren der Mathematischen Wissenschaften 196). Springer, New York.

[5] Billingsley, P. (1968). Convergence of Probability Measures. John Wiley, New York.

[6] Fill, J. A. AND Janson, S. (2000). A characterization of the set of fixed points of the Quicksort transformation. Electron. Commun. Prob. 5, 77-84.

[7] GrüBEL, R. (1998). Hoare's selection algorithm: a Markov chain approach. J. Appl. Prob. 35, 36-45.

[8] Grübel, R. AND RoesLer, U. (1996). Asymptotic distribution theory for Hoare's selection algorithm. Adv. Appl. Prob. 28, 252-269.

[9] Knof, D. And Roesler, U. (2012). The analysis of Find and versions of it. Discrete Math. Theoret. Comput. Sci. 14, 129-154.

[10] Kurtz, T., Lyons, R., Pemantle, R. and Peres, Y. (1997). A conceptual proof of the Kesten-Stigum theorem for multi-type branching processes. In Classical and Modern Branching Processes (IMA Vol. Math. Appl. 84), Springer, New York, pp. 181-185.

[11] Lyons, R., Pemantle, R. And Peres, Y. (1995). Conceptual proofs of $L \log L$ criteria for mean behavior of branching processes. Ann. Prob. 23, 1125-1138.

[12] Martínez, C. (2004). Partial Quicksort. In Proc. 6th Workshop on Algorithm Engineering and Experiments and the First Workshop on Analytic Algorithmics and Combinatorics (New Orleans, January 2004), eds L. Arge, G. F. Italiano and R. Sedgewick, SIAM, pp. 224-228.

[13] Martínez, C. And Rösler, U. (2010). Partial Quicksort and Quickpartitionsort. In 1st International Meeting on Probabilistic, Combinatorial, and Asymptotic Methods in the Analysis of Algorithms (AofA'10; Discrete Math. Theoret. Comput. Sci. AM), Association of Discrete Mathematics and Theoretical Computer Science, Nancy, pp. 505-512.

[14] Meiners, M. and Mentemeier, S. (2017). Solutions to complex smoothing equations. Prob. Theory Relat. Fields 168, 199-268.

[15] Mentemeier, S. (2016). The fixed points of the multivariate smoothing transform. Prob. Theory Relat. Fields 164, 401-458.

[16] NeININGER, R. AND RÜSCHENDORF, L. (2005). Analysis of algorithms by the contraction method: additive and max-recursive equations. In Interacting Stochastic Systems, eds. J.-D. Deuschel and A. Greven, Springer, Berlin, pp. $435-450$.

[17] Neininger, R. and Sulzbach, H. (2015). On a functional contraction method. Ann. Prob. 43, 1777-1822.

[18] Ragab, M. And Roesler, U. (2014). The Quicksort process. Stoch. Process. Appl. 124, 1036-1054. 
[19] Rao, M. M. (1971). Projective limits of probability spaces. J. Multivariate Anal. 1, 28-57.

[20] RösLer, U. (1991). A limit theorem for 'Quicksort'. RAIRO Inf. Théor. Appl. 25, 85-100.

[21] Rösler, U. (1992). A fixed point theorem for distributions. Stoch. Process. Appl. 42, 195-214.

[22] Rösler, U. (1993). The weighted branching process. In Dynamics of Complex and Irregular Systems (Bielefeld, 1991; Bielefeld Encount. Math. Phys. VIII), World Scientific, River Edge, NJ, pp. 154-165.

[23] Rösler, U. (2018). Almost sure convergence to the Quicksort process. To appear in Stoch. Process. Appl.

[24] Rösler, U. AND Rüschendorf, L. (2001). The contraction method for recursive algorithms. Algorithmica 29, 3-33.

[25] Rüschendorf, L. (2006). On the stochastic recursive equations of sum and max type. J. Appl. Prob. 43, 687-703.

\section{S. HALLMANN, Christian-Albrechts-Universität zu Kiel}

Mathematisches Seminar, Christian-Albrechts-Universität zu Kiel, Ludewig-Meyn-Strasse 4, 24098 Kiel, Germany.

\section{U. RÖSLER, Christian-Albrechts-Universität zu Kiel}

Mathematisches Seminar, Christian-Albrechts-Universität zu Kiel, Ludewig-Meyn-Strasse 4, 24098 Kiel, Germany. Email address: roesler@math.uni-kiel.de

M. WNUK, Christian-Albrechts-Universität zu Kiel

Mathematisches Seminar, Christian-Albrechts-Universität zu Kiel, Ludewig-Meyn-Strasse 4, 24098 Kiel, Germany. 